Article

\title{
Promotion of Ionic Conductivity of PEO-Based Solid Electrolyte Using Ultrasonic Vibration
}

\author{
Hui Wang ${ }^{1,2}$, Xiaodong Cui ${ }^{1}{ }^{1}$, Cong Zhang ${ }^{1,2}$, Huang Gao ${ }^{3, *}$, Wei Du $^{1}$ and Yizhe Chen ${ }^{2}$ \\ 1 Hubei Key Laboratory of Advanced Technology for Automotive Components, \\ Wuhan University of Technology, Wuhan 430070, China; huiwang@whut.edu.cn (H.W.); \\ 823416242@whut.edu.cn (X.C.); congzhango@whut.edu.cn (C.Z.); wei.du@whut.edu.cn (W.D.) \\ 2 Hubei Collaborative Innovation Center for Automotive Components Technology, Wuhan 430070, China; \\ yzchen@whut.edu.cn \\ 3 State Key Laboratory of Material Processing and Die \& Mould Technology, \\ School of Materials Science and Engineering, Huazhong University of Science and Technology, \\ Wuhan 430074, China \\ * Correspondence: gaohuang@hust.edu.cn; Tel.: +86-027-87543492; Fax: 86-027-87554405
}

Received: 25 July 2020; Accepted: 18 August 2020; Published: 21 August 2020

\begin{abstract}
All solid-state lithium-ion batteries based on polymer electrolytes have higher safety and energy density, but the low conductivity of lithium ion restricts its application. This study proposes a new method to promote the ionic conductivity of polyethylene oxide (PEO)-based solid electrolytes. In this method, the PEO-based solid electrolyte was first prepared by casting, and then power ultrasound was exerted on the electrolyte by a sandwich structure to modify the electrolyte structure. Through analysis of the performance and microstructure of the electrolyte, it was found that the ultrasonic treatment increased the ionic conductivity by $78 \%$, improved tensile strength and plastic deformation ability, but did not affect the thermal stability and the chemical composition. The ultrasonic vibration, exerting high energy to the solid electrolyte through high-frequency vibration, broke PEO grains and melted them with the frictional heat at boundary. Due to the slight melting and fast solidifying produced by the pulsed ultrasonic treatment, the crystallization was suppressed. The crystallinity was thus reduced by $6.2 \%$, which increased the migration channels of lithium ions and reduced the tortuosity effect. Furthermore, the ultrasonic vibration compressed the electrolyte to produce plastic flow of the material, which made the electrolyte structure more compact. The density of ethylene oxide (EO) units thus increased in the amorphous phase, providing multiple electron-donor coordination sites for the $\mathrm{Li}^{+}$. The hopping distance of the ion between donors decreased, which also facilitated the migration. In addition, the mechanical performance of the electrolyte membrane improved. This study provides a reference for the improvement of polymer based all-solid-state batteries.
\end{abstract}

Keywords: solid electrolyte; ultrasonic vibration; polyethylene oxide; ionic conductivity; improvement; crystallinity

\section{Introduction}

In recent years, with the advancement of new energy vehicles, the market has put forward new requirements for batteries. As the core component of electric vehicles, power batteries will encounter new opportunities and challenges. In the future, the energy density of the power batteries of electric vehicles must reach at least $500 \mathrm{~W} \cdot \mathrm{h} / \mathrm{kg}$, but the energy density of large-scale practical lithium-ion batteries is far from this goal. Compared with traditional lithium-ion batteries, the all-solid-state lithium ion battery has better safety and higher energy density [1], and are expected to be used in electric vehicles, and therefore they have attracted widespread attention. 
Compared with the liquid electrolyte, the solid electrolyte has poor conductivity of lithium ions, and the conductivity is usually only $10^{-5} \mathrm{~S} / \mathrm{cm}$, while the liquid electrolyte ion conductivity can reach $10^{-2} \mathrm{~S} / \mathrm{cm}$. The migration of lithium ions in the solid electrolyte is weaker than that in the liquid electrolyte [2-7]. The polyethylene oxide (PEO)-based solid electrolyte is the most widely studied in polymer based all-solid-state lithium batteries. PEO is a semi-crystalline polymer. Generally, lithium ion transport in a PEO-based solid electrolyte is derived from segmental movement that can be accelerated in the amorphous PEO compared with that in the crystalline PEO [8]. Therefore, an effective way to increase the ionic conductivity of the PEO-based electrolyte is to reduce its crystallinity and increase the proportion of the amorphous phase.

At present, the main methods for reducing the crystallinity include plasticizers, ionic liquids, inorganic fillers and copolymers. Chintapalli $S$ et al. [9] studied the effect of the plasticizer ethylene carbonate (EC) and propylene carbonate (PC) on the high molecular weight $\mathrm{PEO}_{9}-\mathrm{LiCF}_{3}-\mathrm{SO}_{3}$ system, and found that the addition of the plasticizer significantly improved the ionic conductivity of the electrolyte at room temperature. Kumar et al. [10] added 1-Ethyl-3-methy limidazolium trifluoromethane-sulfonate (EMITF) ionic liquid to the PEO-based electrolyte system, and found that the organic cation $\mathrm{EMI}^{+}$can interact with the PEO segment, increase the amorphous phase ratio, and improve the lithium ion transport capacity. Scrosati et al. [11] studied the influence of different inorganic ceramic fillers on the performance of the solid polymer electrolyte $\mathrm{PEO}-\mathrm{LiCF}_{3} \mathrm{SO}_{3}$ system, and found that the addition of inorganic ceramic fillers can effectively improve the interface stability of the electrolyte and the lithium metal electrode, and enhance the electrolyte's overall transport properties. Tan SM et al. [12] studied the effect of nano- $\mathrm{MnO}_{2}$ filler on the ionic conductivity of the plasticized polymer electrolyte PMMA-PEO- $\mathrm{LiClO}_{4}$-EC system. By optimizing the composition of the polymer, salt, plasticizer and filler, the ionic conductivity of the electrolyte can reach $10^{-3} \mathrm{~S} / \mathrm{cm}$. K. Nairn et al. [13] added the ceramic solid electrolyte $\mathrm{Li}_{1.3} \mathrm{Al}_{0.3} \mathrm{Ti}_{1.7}\left(\mathrm{PO}_{4}\right)_{3}$ into a pure amorphous copolymer electrolyte polyethylene glycol(PEG)- $\mathrm{LiCF}_{3} \mathrm{SO}_{3}$ to prepare an organic-inorganic solid electrolyte. When the inorganic filler was $66 \mathrm{wt} . \%$ and the temperature was $40{ }^{\circ} \mathrm{C}$, the composite electrolyte had an ionic conductivity of $(1.9 \pm 0.2) \times 10^{-4} \mathrm{~S} / \mathrm{cm}$. Xiao-Yuan Yu et al. [14] prepared a new type of $\mathrm{PEO} /$ poly propylene carbonate (PPC) solid electrolyte by blending $\mathrm{PEO}$ with poly propylene carbonate (PPC), and found that the addition of PPC can effectively reduce the glass transition temperature $\mathrm{Tg}$ and crystallinity of the PEO and improve the ionic conductivity of the electrolyte. Niitani T et al. [15] applied a block copolymer consisting of $\mathrm{PEO}$ and polystyrene (PS) to prepare a $\mathrm{LiClO}_{4}$-contained solid polymer electrolyte with high ionic conductivity at room temperature.

Power ultrasound, an interesting processing method for polymer materials, produces thermal and mechanical changes in the materials by low-amplitude and high-frequency vibration [16,17]. Related research has shown that the ultrasound can produce high-frequency vibration effects on the blend melt, and can effectively promote the mutual diffusion and fusion of molecular chains of different polymers. Ultrasonic treatment enhances the interaction between molecules, promotes chemical bonds between different polymers without using any chemical catalysts, induces in-situ copolymer at the interface of polymer blends, causes micromechanical mixing, and improves the interface bond of phases [18,19]. Madhusudan Bera et al. [20] found that the ultrasound promoted the formation of covalent bonds, thereby producing macromolecular structures. The ultrasonic frequency was $15-120 \mathrm{kHz}$ in actual use. The mechanical vibration preferentially generated heat at the interface through the friction effect, and therefore the interface bonding strength can reach $100 \%$ of the matrix [21]. Fernandez Villegas et al. [22] proved that the high-frequency vibration of the ultrasound can generate ultra-short heating at the interface, causing the thermoplastics to melt, and thus two pieces can be joined together under pressure. Chu et al. [23] used differential scanning calorimetry (DSC) to study the crystallinity of samples prepared by the ultrasonic vibration-assisted automated fiber placement (UAFP) and hot pressing. The results showed that the crystallinity of the UAFP sample was only $38.5 \%$, while the crystallinity of the hot-pressed sample was $49.2 \%$. 
In published reports, there is no study on the application of ultrasonic vibration for the improvement of the ionic conductivity of the solid polymer electrolyte. If the polymer electrolyte is subjected to ultrasound, high-frequency vibration breaks the electrolyte grains, increasing the ratio of the amorphous phase, and thereby improves the lithium ion conductivity. In this study, a PEO-based solid electrolyte was first prepared by casting, and then power ultrasound was exerted on the electrolyte to modify the structure. Through tests on the structure and performance of the electrolyte, the effect of the ultrasonic vibration was analyzed.

This paper is organized as follows. Section 2 describes the materials and ultrasonic treatment method. Section 3 gives the results and discussion. Finally, Section 4 summarizes our conclusions.

\section{Materials and Methods}

\subsection{Materials}

The resin-matrix material for the solid polymer electrolyte was PEO (Mw $=10^{6}$; Ryoji Organic Chemical, Shanghai, China). The melting temperature is $65^{\circ} \mathrm{C}$, and the density is $1.15-1.22 \mathrm{~g} / \mathrm{cm}^{3}$. The structural formula is shown in Figure 1a. The ether group $\mathrm{C}-\mathrm{O}-\mathrm{C}$ is the function group, which provides electron-donor coordination site for the $\mathrm{Li}^{+}$ion. In addition, the end group $\mathrm{C}-\mathrm{OH}$ is also involved in the molecule. Acetonitrile (ACN, 99.8\%; Tianjin Beilian Fine Chemicals Development Co., Ltd., Tianjin, China), and lithium bis ((trifluoromethyl) sulfonyl) azanide (LiTFSI, 99\%; Shanghai Aichun Biological Technology, Shanghai, China) were used for the solvent and lithium salt, respectively. The density of the lithium salt is $1.334 \mathrm{~g} / \mathrm{cm}^{3}$. The structural formulas are shown in Figure 1. The group $\mathrm{C} \equiv \mathrm{N}$ is the characteristic group, and can be used to identify the presence of the acetonitrile. The sulfonyl group $\mathrm{O}=\mathrm{S}=\mathrm{O}$ and the trifluoromethyl group $\mathrm{CF}_{3}$ are both characteristic groups of the salt LiTFSI.

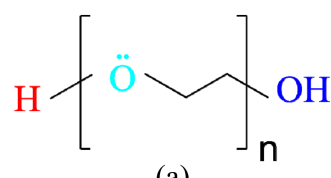

(a)<smiles>CC#N</smiles>

(b)

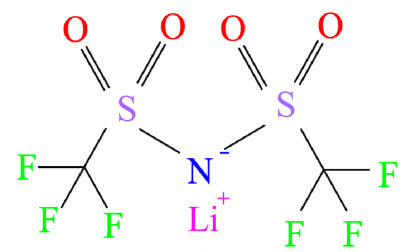

(c)

Figure 1. Structural formulas of (a) polyethylene oxide (PEO), (b) acetonitrile, and (c) lithium bis ((trifluoromethyl) sulfonyl) azanide (LiTFSI).

\subsection{Experimental Method}

\subsubsection{Preparation of Polymer Solid Electrolyte Membrane}

The preparation process of the solid electrolyte membrane is shown in Figure 2. The PEO powder and LiTFSI were dried in a vacuum oven at $60{ }^{\circ} \mathrm{C}$ for $24 \mathrm{~h}$ to remove residual moisture, and then these materials were put into a glove box (Super, Mikrouna, Shanghai, China). To obtain an excellent solid polymer electrolyte (SPE), the ratio of ethylene oxide (EO): $\mathrm{Li}=18: 1$ is required, and thus $1.25 \mathrm{~g}$ PEO and $0.25 \mathrm{~g}$ LiTFSI were weighed with a precision electronic balance (8086, Dongguan DiHeng instrument, Dongguan, China). Then, $30 \mathrm{~mL}$ acetonitrile was poured into a clean, dry beaker. Firstly, the LiTFSI was added to the beaker, and the solution was stirred magnetically with a magnetic stirrer (ZGCJ-3A, Shanghai ZiGui instrument, Shanghai, China). After the LiTFSI was dissolved completely, the PEO was added slowly. Subsequently, the beaker was placed on the magnetic stirrer, with which the solution was stirred for $12 \mathrm{~h}$. After that, the solution appeared transparent and homogeneous. The solution was quickly poured into a polytetrafluoroethylene (PTFE) mold to form a membrane. The solution flowed naturally in the cavity until it was filled up. The mold was kept in the glove box for $3 \mathrm{~h}$ to eliminate most acetonitrile solvent, and then the mold was dried in a vacuum oven (DZF-6020B, Changzhou EnPei instrument Co. Ltd., Changzhou, China) at $45^{\circ} \mathrm{C}$ for 
$24 \mathrm{~h}$. The electrolyte membrane was removed with a tweezer, and was cut into circular pieces of 16 $\mathrm{mm}$ in diameter, as shown in Figure 3. The pieces were kept in the glove box for later use.

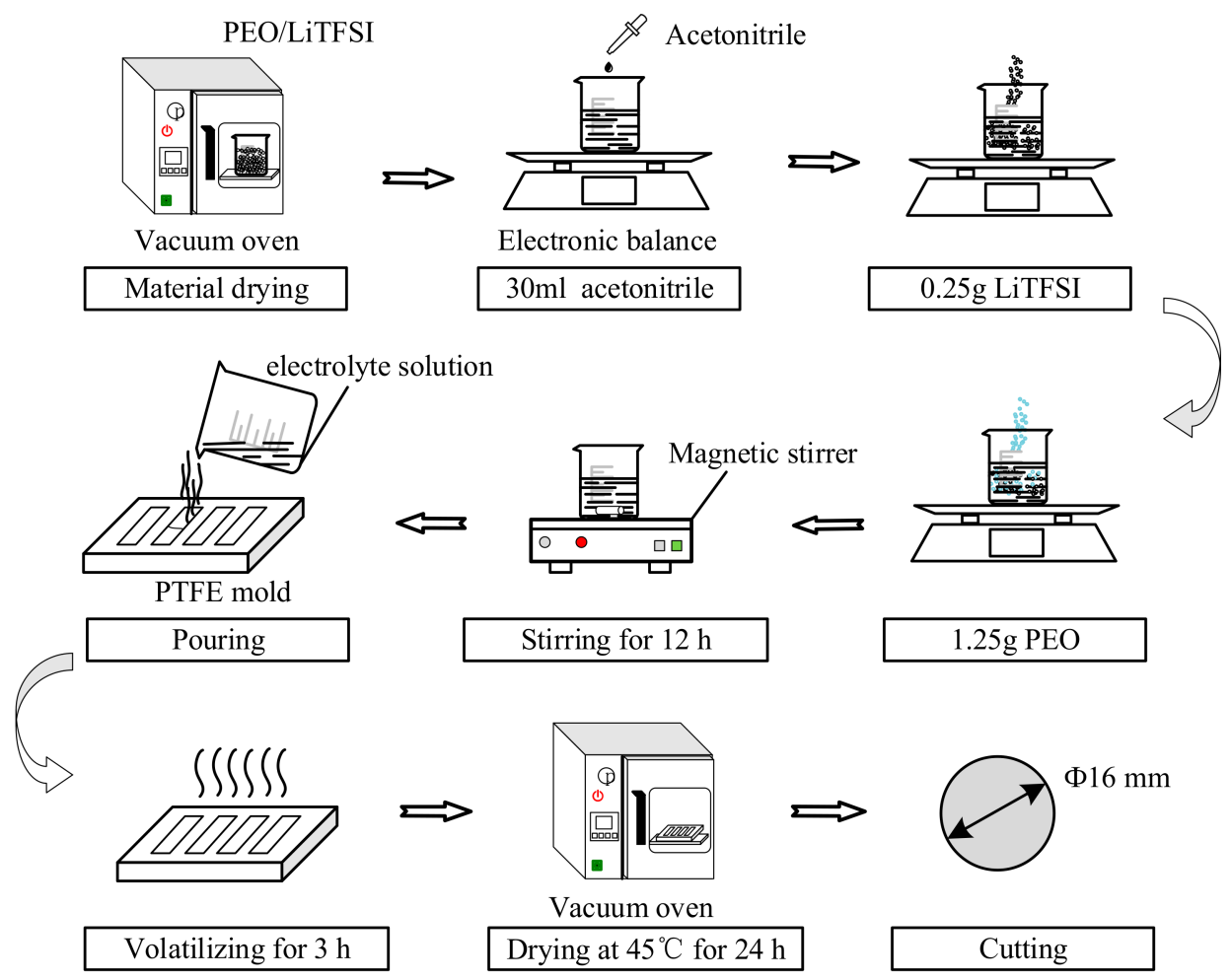

Figure 2. Preparation process of the solid electrolyte membrane.

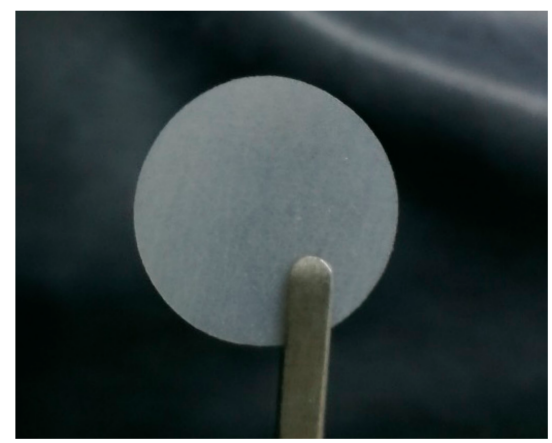

Figure 3. Cut pieces of the electrolyte.

\subsubsection{Ultrasonic Treatment of the Solid Electrolyte}

In this experiment, the Taiwan MAXWIDE ${ }^{\circledR}$ ME-1800 ultrasonic platform was applied, as shown in Figure 4. The device is a vertical structure, and is mainly composed of an ultrasonic generator, an ultrasonic transducer, a horn and a sonotrode. The transducer, horn and sonotrode are mounted on a pneumatic press machine, and can be moved up and down along a vertical guide-bar. The maximum pressure is $0.8 \mathrm{MPa}$. When the device is in operation, the ultrasonic generator converts civilian electricity of $220 \mathrm{~V}$ and $50 \mathrm{~Hz}$ into high-frequency alternating current (AC) electrical signals to match with the ultrasonic transducer. Then the transducer converts the high-frequency electrical signals into mechanical vibration. The horn, made of steel, amplifies the vibration. The sonotrode is of a cylinder type, which is made of 7075 aviation aluminum alloy. It is tightly fixed to the horn to concentrate the energy of the ultrasonic system on a relatively small area. 


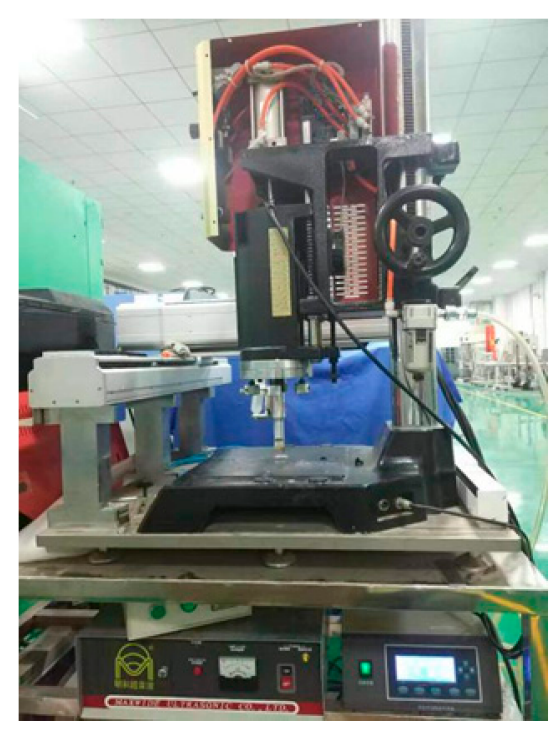

Figure 4. Experiment platform for the ultrasonic treatment.

Ultrasonic vibration was applied to modify the microstructure of the electrolyte. As shown in Figure 5, we used a sandwich structure, which included a cover plate, the electrolyte, and a base plate. The base plate, made of 7075 aluminum alloy, was fixed on the experimental platform to prevent the tearing of the electrolyte membrane due to the horizontal slip during the ultrasonic treatment. In order to apply uniform force on the electrolyte, a smooth plate was covered on the electrolyte membrane. The cover plate should not be too thick, otherwise it will affect the transmission of the ultrasonic vibration. It should not be too thin, otherwise the plate deforms easily due to the low rigidity during the application of the vibration, leading to inhomogeneous force on electrolyte membrane. In this study, a $20 \times 20 \times 2 \mathrm{~mm}$ carbon fiber reinforced plastics (CFRP) laminate was used as the cover plate. According to orthogonal experimental design, the ultrasonic frequency used was $15 \mathrm{kHz}$, the pressure was $0.24 \mathrm{MPa}$, the amplitude was $40 \mu \mathrm{m}$, and the vibration time was 32 s. During the vibration, an intermittent mode was applied to avoid electrolyte ablation caused by the ultrasonic overheating. In one cycle of the mode, the ultrasonic operated for $1 \mathrm{~s}$, and suspended for $3 \mathrm{~s}$. The solid electrolyte membrane without the ultrasonic treatment was recorded as Group A, and the solid electrolyte film treated by the ultrasonic vibration was recorded as Group B.

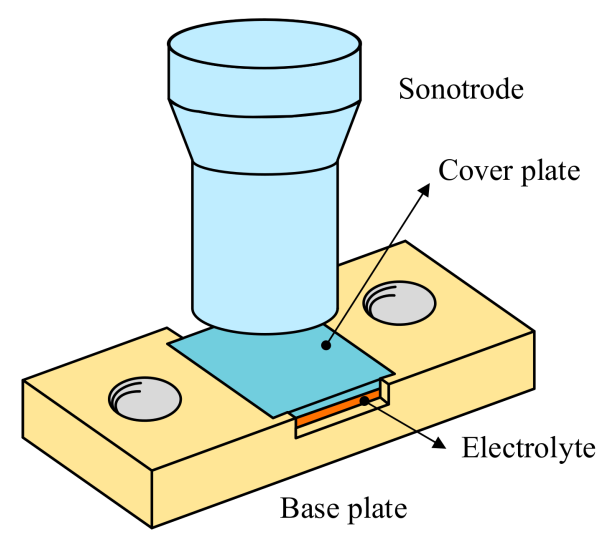

Figure 5. Schematic of the ultrasonic treatment. 


\subsection{Characterization}

\subsubsection{AC Impedance}

The SPE was measured by an electrochemical workstation, and the ionic conductivity was then obtained by the analysis of the measured AC impedance spectra. The test instrument used was the CHI760E electrochemical workstation (Shanghai Chenhua Instrument, Shanghai, China). The structure of stainless steel (SS)/SPE/SS was applied for the test. The sweep frequency range was $0.1 \sim 10^{6} \mathrm{~Hz}$, and the test temperature was from $25^{\circ} \mathrm{C}$ to $70^{\circ} \mathrm{C}$.

\subsubsection{Direct Current (DC) Polarization}

The conductivity was analyzed by the DC polarization method. A good solid electrolyte should have enough electronic resistivity, otherwise it will short circuit inside the battery. The test instrument used was the BTS4000 analyzer (NEWARE, Shenzhen, China). The structure of SS/SPE/SS was applied. The test voltage was $3 \mathrm{~V}$, and the test time was $320 \mathrm{~min}$.

\subsubsection{Linear Sweep Voltammetry (LSV)}

The electrochemical window of the electrolyte was determined by the LSV test. The test instrument used was the CHI760E electrochemical workstation. The structure of Li/SPE/SS was applied. The initial voltage was $2.9 \mathrm{~V}$, the termination voltage was $6 \mathrm{~V}$, and the scanning frequency was $5 \times 10^{4} \mathrm{~Hz}$.

\subsubsection{Scanning Electron Microscope (SEM)}

The microstructure of the electrolyte was observed by the JEM-7500F electron microscope (JEOL, Tokyo, Japan). Before the observation, the electrolyte was fractured in liquid nitrogen. After platinum coating, the fracture surface was observed.

\subsubsection{Mechanical Performance Test}

Mechanical property is an important index to evaluate the solid electrolyte. Excellent mechanical property can effectively inhibit the growth of lithium dendrites and ensure safety during cycles. In this study, the Instron1341 electro-hydraulic servo testing machine (Instron, High Wycombe, UK) was used to test the mechanical property of the solid electrolyte.

\subsubsection{Thermogravimetric Analysis (TGA)}

Thermogravimetric analysis was used to test the thermal stability of the electrolyte. The test instrument used was the TGA 5500 analyzer (TA, New Castle, DE, USA). The sample used for the analysis was cut into $3 \mathrm{~mm}^{2}$. The temperature rise rate was set as $10^{\circ} \mathrm{C} / \mathrm{min}$, and the temperature range was $0 \sim 800^{\circ} \mathrm{C}$.

\subsubsection{Fourier Transform Infrared (FTIR) Spectroscopy}

In this study, the Nicolet 6700 infrared spectrometer (Thermo Fisher Scientific, Waltham, MA, USA) was used for the test of FTIR spectra. The wave number was from $65 \mathrm{~cm}^{-1}$ to $4000 \mathrm{~cm}^{-1}$.

\subsubsection{X-ray Diffraction (XRD)}

An X-ray diffractometer was used to examine the crystallinity, and the XRD pattern was analyzed to calculate the change in the crystallinity of the electrolytes with and without the ultrasonic treatment. The test instrument used was the Empyrean X-ray diffractometer (PANalytical, Almelo, Netherlands). The tube voltage was $40 \mathrm{kV}$, the scanning angle range was $10^{\circ} \sim 50^{\circ}$, and the scanning step size was $2 \% \mathrm{~s}$. 


\section{Results}

\subsection{AC Impedance and Ionic Conductivity}

The AC impedance spectra tested by the electrochemical workstation are shown in Figure 6. With the increase in the temperature, the impedance curves did not show a complete semicircle at low impedance region (high frequency), but gradually approached the oblique line at the diffusion region (low frequency). The rise in temperature promoted movement of the PEO chains, leading to the dominance of $\mathrm{Li}^{+}$diffusion in the electrolyte. The impedance decreased gradually with the temperature rise, and the ionic conductivity increased. The melting temperature of the PEO is 65 ${ }^{\circ} \mathrm{C}$. When the temperature was $70{ }^{\circ} \mathrm{C}$, the electrolyte changed from the solid state to the molten one. PEO chains were released, and the impedance of the electrolyte was thus significantly reduced.

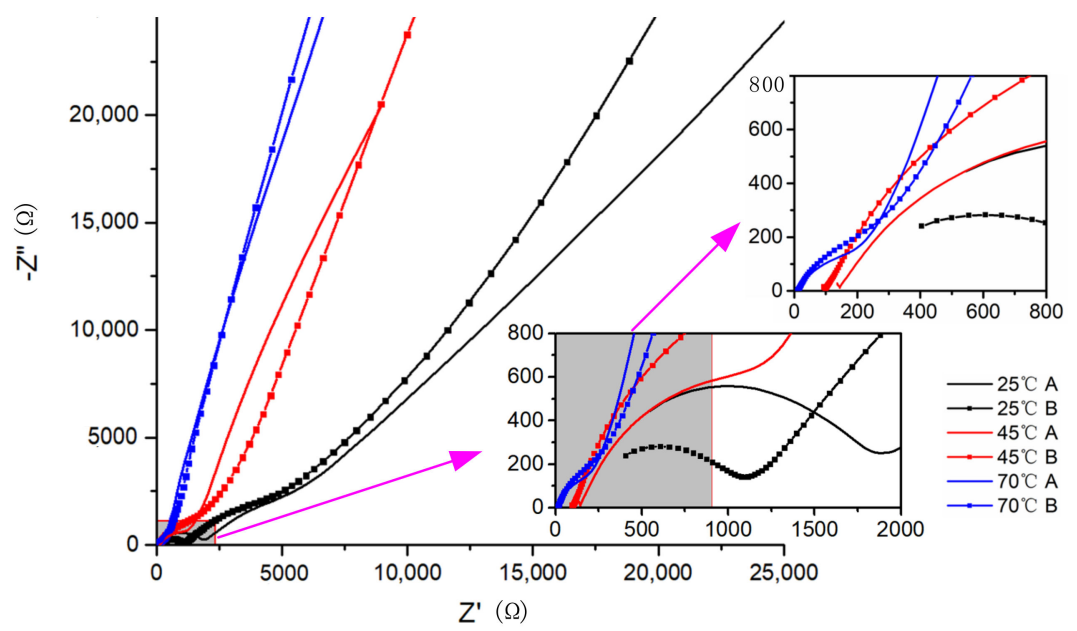

Figure 6. Alternating current $(A C)$ impedance tests at different temperatures, where Group A is non-ultrasonic treated and Group B is ultrasonic treated.

The ionic conductivity was derived from the AC impedance spectra. The ionic conductivity can be calculated by:

$$
\sigma=\frac{L}{R \times S}
$$

where, $R$ is the resistivity of the electrolyte membrane, $S$ is the surface area, and $L$ is the thickness. The membrane thickness was measured with a micrometer.

The calculated ionic conductivity of the electrolytes from Group A and Group B is listed in Table 1.

Table 1. Impedance and ionic conductivity of the electrolytes.

\begin{tabular}{ccccc}
\hline & \multicolumn{2}{c}{ Impedance $(\Omega)$} & \multicolumn{2}{c}{ Ionic Conductivity $(\mathrm{S} / \mathrm{cm})$} \\
\hline & $25^{\circ} \mathrm{C}$ & $45^{\circ} \mathrm{C}$ & $25^{\circ} \mathrm{C}$ & $45^{\circ} \mathrm{C}$ \\
\hline $\mathrm{A}$ & 1960 & 144 & $1.8 \times 10^{-6}$ & $2.4 \times 10^{-5}$ \\
$\mathrm{~B}$ & 1100 & 99 & $3.2 \times 10^{-6}$ & $3.6 \times 10^{-5}$ \\
\hline
\end{tabular}

At $25{ }^{\circ} \mathrm{C}$, the impedance of the electrolyte decreased from $1960 \Omega$ to $1100 \Omega$ due to the ultrasonic treatment, with a decrease of $44 \%$. The corresponding ionic conductivity increased from $1.8 \times 10^{-6}$ $\mathrm{S} / \mathrm{cm}$ to $3.2 \times 10^{-6} \mathrm{~S} / \mathrm{cm}$ at $25{ }^{\circ} \mathrm{C}$, and it was significantly improved by $78 \%$ with the ultrasonic treatment. At $45{ }^{\circ} \mathrm{C}$, the ionic conductivity was also significantly improved due to the ultrasonic treatment. At $70{ }^{\circ} \mathrm{C}$, the PEO-based electrolyte was molten. The chains were released completely, and thus the ultrasonic treatment had little effect on the electrolyte at this temperature, as shown in Figure 6. After the molten electrolyte was cooled to room temperature, it lost the increased 
conductivity completely. The impedance was tested for the annealed electrolyte of Group B, and the spectrum is shown in Figure 7. For comparison, the impedance spectrum of the electrolyte without the ultrasonic treatment (Group A) was also presented in the figure. The impedance measured $1942 \Omega$, and the corresponding ionic conductivity was $1.82 \times 10^{-6} \mathrm{~S} / \mathrm{cm}$, which was similar to that of the electrolyte without the ultrasonic treatment (Group A of Table 1).

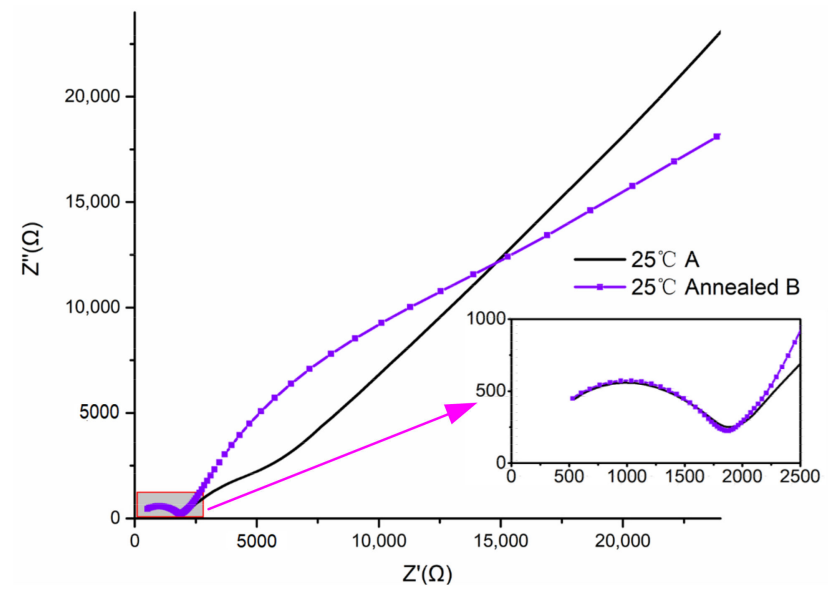

Figure 7. AC impedance test at $25^{\circ} \mathrm{C}$ for the annealed electrolyte of Group B.

\subsection{Electronic Conductivity}

The DC polarization test results of the electrolytes are shown in Figure 8. It can be seen that the polarization current of the electrolyte without the ultrasonic treatment (Group A) was 0.103 $\mathrm{mA}$, and the steady current was $0.0012 \mathrm{~mA}$. The polarization current of the electrolyte treated by the ultrasonic vibration (Group B) was $0.0573 \mathrm{~mA}$, and the steady current was $0.0012 \mathrm{~mA}$. The results suggest that the ultrasonic treatment did not obviously change the electronic conductivity. The prepared electrolytes both had enough electronic resistivity.

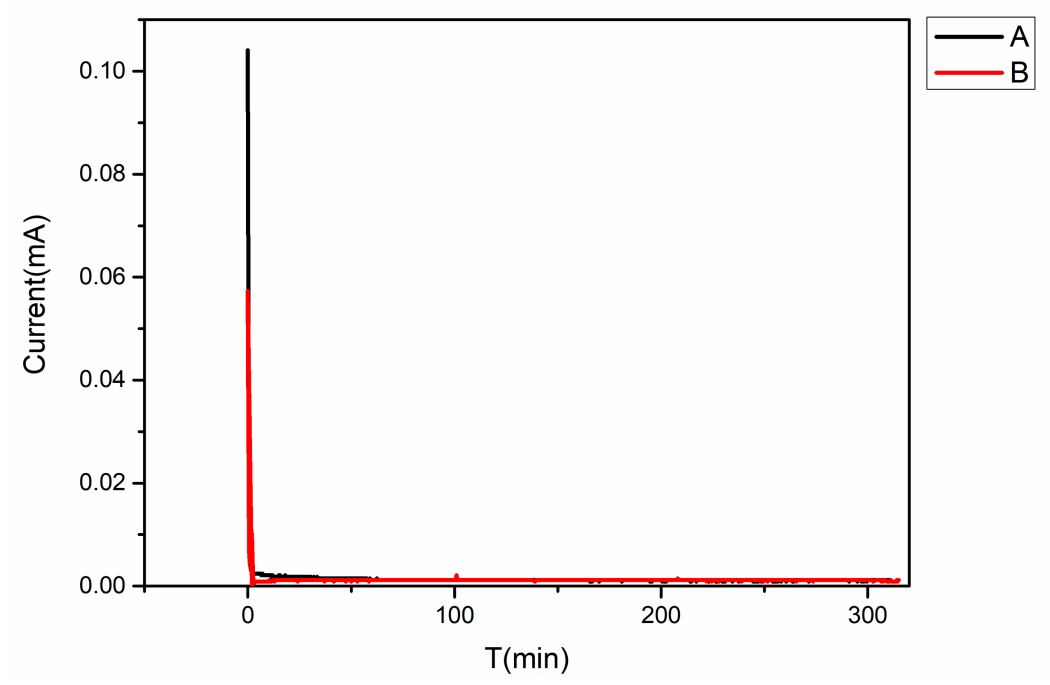

Figure 8. Direct Current (DC) polarization test results of the PEO-based solid electrolyte.

\subsection{Electrochemical Window}

Electrolytes with a wide electrochemical window can match more types of electrode material. Therefore, a wide and stable electrochemical window is necessary for the electrolytes prepared. Currently, the electrochemical window of commercial lithium-ion batteries is $3 \sim 4 \mathrm{~V}$. The electrochemical 
window of the prepared PEO-based electrolyte was tested by the LSV test, and the results are shown in Figure 9. The test result of Group A was similar to that of Group B, so we just presented that of Group $\mathrm{B}$ to illustrate the electrochemical window of the prepared electrolyte membrane.

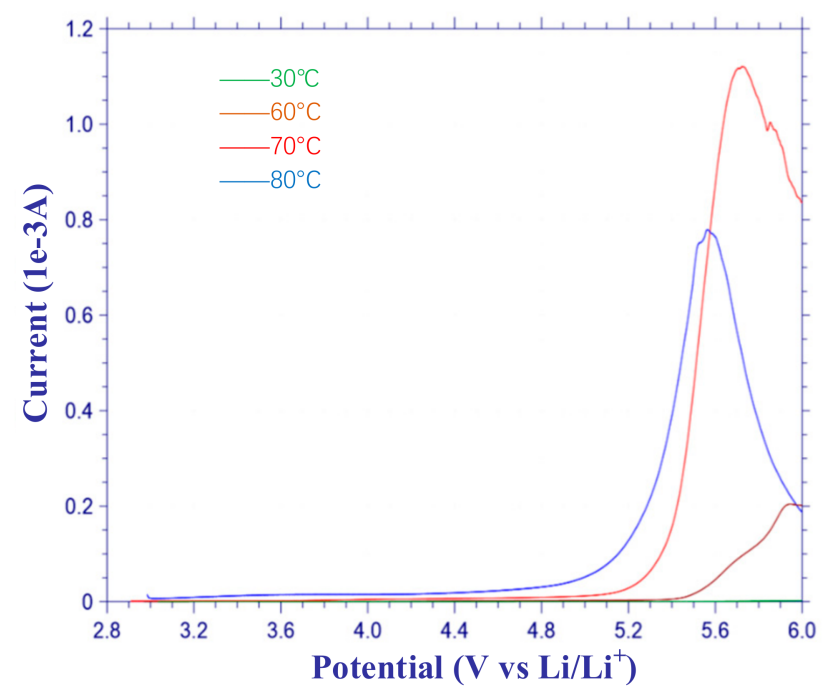

Figure 9. Linear sweep voltammetry (LSV) test results.

As shown in Figure 9, the voltage threshold exceeded $6 \mathrm{~V}$ at $30^{\circ} \mathrm{C}$. As the voltage increased, the current of the electrolyte membrane did not change significantly until the voltage exceeded $5.0 \mathrm{~V}$ at $70{ }^{\circ} \mathrm{C}$. With the temperature rising, the electrochemical window narrowed, and reached a minimum value of $4.8 \mathrm{~V}$ at $80^{\circ} \mathrm{C}$. This demonstrates that the prepared PEO-LiTFSI electrolyte membrane had a wide electrochemical window, which can satisfy the charge/discharge voltage of 2 3.6 V for lithium-ion batteries.

\subsection{Mechanical Strength}

The mechanical strength of the electrolyte membrane is very important for all-solid-state lithium batteries. Sufficient mechanical strength can prevent short-circuiting caused by the penetration of lithium dendrite into the electrolyte membrane. The Instron134 material testing machine was used to test the tensile properties. The size of the sample is shown in Figure 10. The total length was 150 $\mathrm{mm}$, the width was $20 \mathrm{~mm}$, and the gauge length was $50 \mathrm{~mm}$. The test was conducted at a speed of $5 \mathrm{~mm} / \mathrm{min}$. Two pads were added in the fixture area according to the standard of GB/T1040.3-2006, and the size was $25 \times 20 \times 1.5 \mathrm{~mm}$.

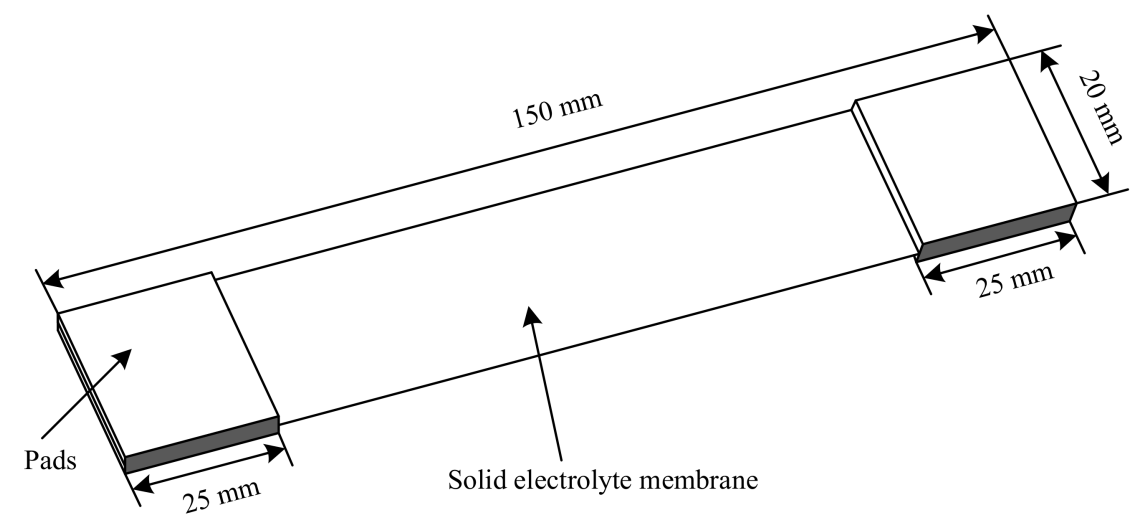

Figure 10. Sample of the electrolyte for the tensile test. 
As shown in Figure 11, when the strain was below 20\%, the tensile stress increased proportionally with the strain, indicating an elastic deformation. Furthermore, the curve of Group B had a significantly lower slope than that of Group A, which shows that the modulus of the electrolyte was decreased by the ultrasonic treatment. When the strain continued to increase, the stress increase rate slowed down, and the material underwent a plastic deformation. When the strain of Group A and Group B exceeded $600 \%$ and $800 \%$, respectively, the stress rose rapidly. The polymer orientation of the electrolyte membranes increased, enhancing the material. Without the ultrasonic treatment (Group A), the electrolyte snapped at the strain of $1087 \%$, and the tensile strength was $2.47 \mathrm{MPa}$. For the electrolyte treated by the ultrasonic process, the tensile failure occurred at the strain of $1734 \%$, and the tensile strength was $3.06 \mathrm{MPa}$. The tensile strain and strength of the electrolyte was improved by the ultrasonic treatment. However, the yield strength of Group A was $0.6 \mathrm{MPa}$, while that of Group B was $0.49 \mathrm{MPa}$. The yield strength and elastic modulus of the electrolyte were decreased by the ultrasonic treatment.

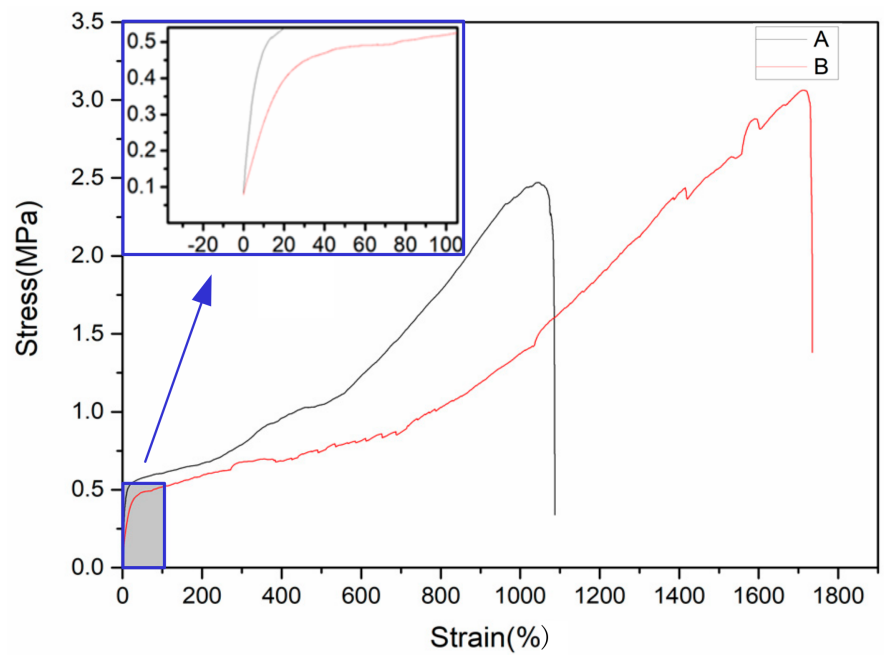

Figure 11. Stress-strain curves of the PEO-based electrolytes.

\subsection{Morphology}

The microstructure of the electrolyte membranes with and without the ultrasonic treatment was characterized by SEM. Figure 12 shows the cross-sectional morphology of the electrolytes. As shown in this figure, no obvious crack was observed inside the electrolyte membranes, indicating good toughness and low internal residual stress. Microparticles were observed inside the electrolytes, and were the undissolved lithium salt. As displayed in Figure 12, there were few particles, indicating that the prepared electrolytes were essentially homogeneous. Furthermore, the LiTFSI salt was evenly distributed, and thus the ratio of $\mathrm{EO}: \mathrm{Li}=18: 1$ in the electrolytes was ensured, which was beneficial to the transport of $\mathrm{Li}^{+}$. Comparing Figure $12 \mathrm{a}$ with $\mathrm{b}$, material reflow lines were observed in the electrolyte membrane treated by the ultrasonic process. With the ultrasonic treatment, heat was generated in the electrolyte membrane. The ultrasonic vibration, increasing mobility of the polymer chains, was also beneficial to the material flow. At the same time, the membrane was compressed in the ultrasonic process, and thus the plastic flow of the material was produced, which modified the structure of the material. The solid electrolyte became tighter. In addition, we also measured the apparent density of the electrolyte. The density was $1.018 \mathrm{~g} / \mathrm{cm}^{3}$ for the electrolyte of Group A, and was $1.136 \mathrm{~g} / \mathrm{cm}^{3}$ for Group B. During the measurement, circular pieces of $10 \mathrm{~mm}$ in diameter were applied. The measured thickness was $0.081 \mathrm{~mm}$ and $0.072 \mathrm{~mm}$ for Group A and Group B, respectively, and the corresponding weight was $6.47 \mathrm{mg}$ and $6.42 \mathrm{mg}$ for them. From the measured density, the electrolyte with the ultrasonic treatment was tighter than that without the treatment. Therefore, the ion migration rate was increased by the ultrasonic treatment, and the mechanical strength was also improved. 


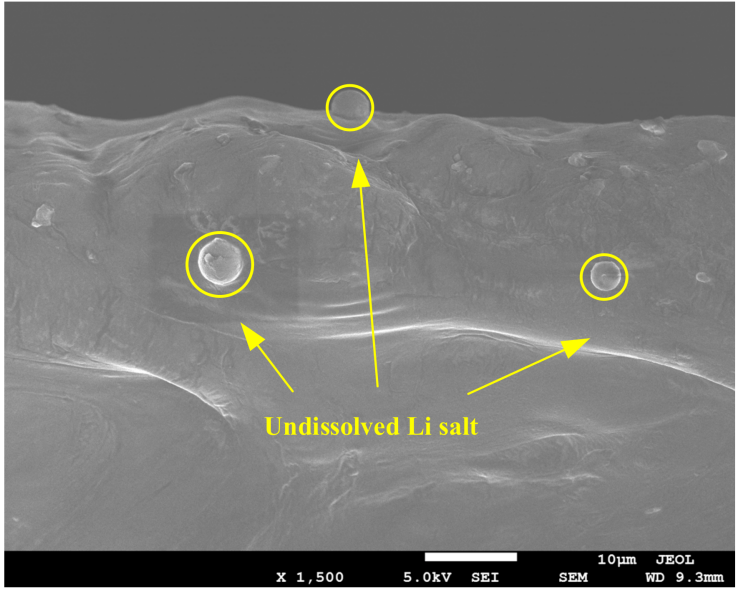

(a)

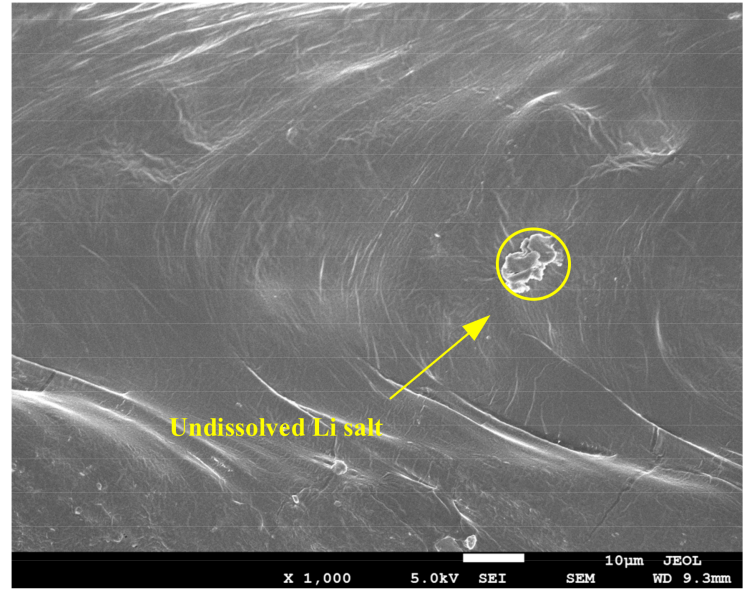

(b)

Figure 12. SEM observation of the electrolyte: (a) from Group A, and (b) from Group B.

\subsection{Thermal Stability}

The TGA test results are shown in Figure 13. The solid electrolyte membranes of both groups were thermally degraded within a temperature range of $300 \sim 400{ }^{\circ} \mathrm{C}$, and the weight was lost primarily in one stage. The high temperature caused the degradation of PEO chains in the matrix. According to the FTIR spectrum analysis in Section 3.7, the initial mass loss was caused by the moisture absorption of the samples during the test. From the test data, the water loss was $1.428 \times 10^{-5}$ mol for Group A, and $1.422 \times 10^{-5} \mathrm{~mol}$ for Group B. The temperature of $150{ }^{\circ} \mathrm{C}$ was taken as the end point of the water lost. Furthermore, both samples began to decompose near $320^{\circ} \mathrm{C}$. This demonstrates that the ultrasonic treatment did not affect the thermal stability of the electrolyte membranes.

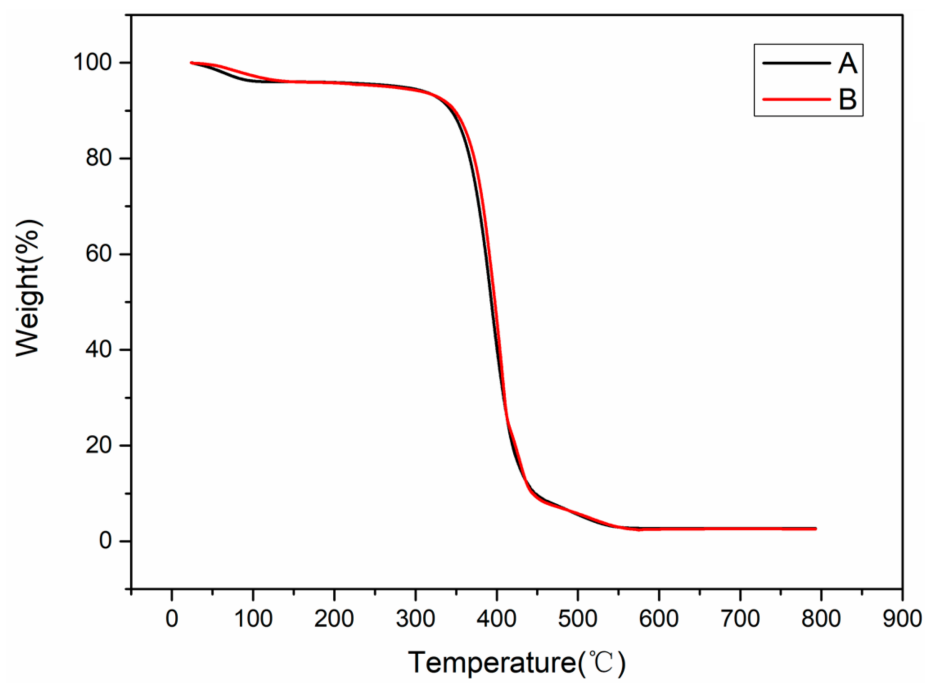

Figure 13. TGA results of the electrolytes.

\subsection{FTIR Analysis}

The infrared spectra of the PEO-based electrolytes are shown in Figure 14. As displayed in this figure, the characteristic peaks were observed at $3470 \mathrm{~cm}^{-1}$ and $1670 \mathrm{~cm}^{-1}$ due to the water, which might be attributed to the absorbed moisture in the samples. Furthermore, no peak was observed near $2250 \mathrm{~cm}^{-1}$, the characteristic peak of $\mathrm{C} \equiv \mathrm{N}$ in the acetonitrile, indicating that the residual solvent 
was not detected in the electrolyte. Therefore, the mass loss below $100^{\circ} \mathrm{C}$ in Section 3.6 was due to evaporation of the absorbed moisture during the test.

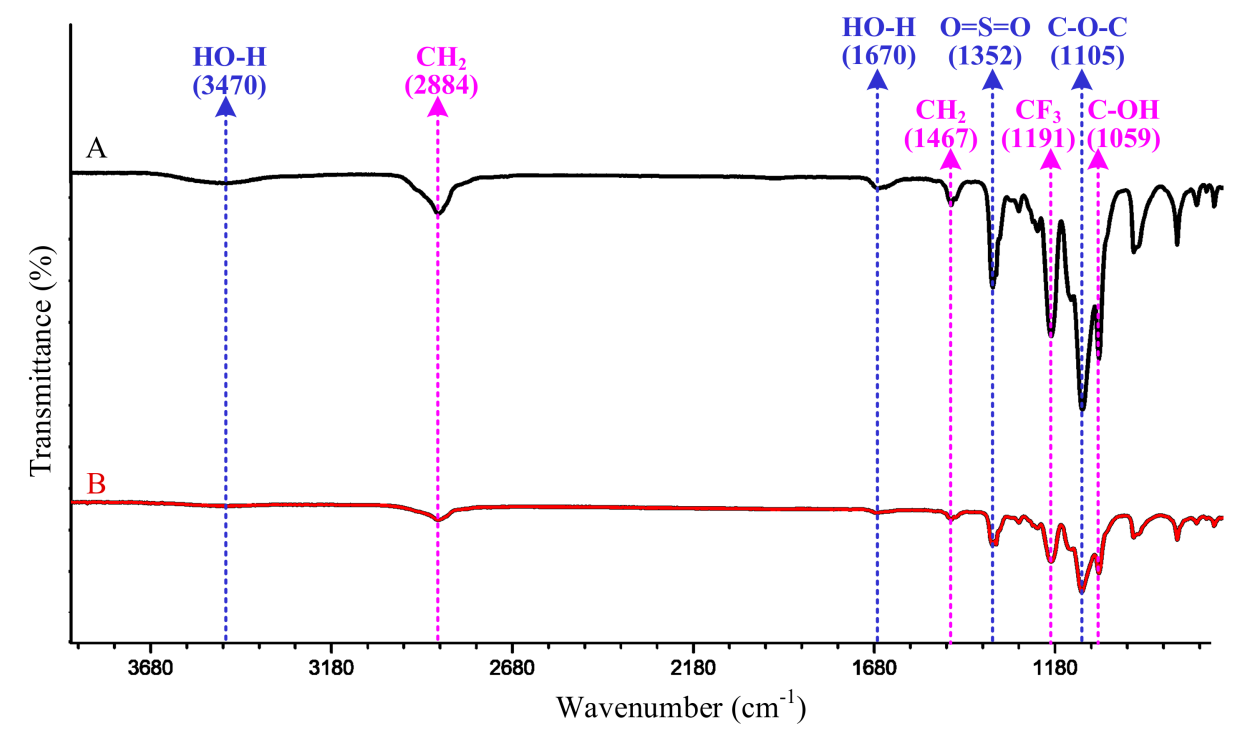

Figure 14. FTIR test results of the electrolytes.

Both spectra exhibited two peaks at $2884 \mathrm{~cm}^{-1}$ and $1467 \mathrm{~cm}^{-1}$, which were due to the stretching and bending vibration of $\mathrm{CH}_{2}$ in the PEO chains. The peak at $1352 \mathrm{~cm}^{-1}$ was due to the stretching vibration of the $\mathrm{O}=\mathrm{S}=\mathrm{O}$ group, while that at $1191 \mathrm{~cm}^{-1}$ was due to the stretching vibration of the $-\mathrm{CF}_{3}$ group. The two peaks were characteristic peaks of the LiTFSI salt. Ether group $\mathrm{C}-\mathrm{O}-\mathrm{C}$ in the PEO chains was identified at the characteristic peak of $1105 \mathrm{~cm}^{-1}$. By providing the electron-donor coordination site for the ion, the group $\mathrm{C}-\mathrm{O}-\mathrm{C}$ served as the function group for the $\mathrm{Li}^{+}$ion transport because of the $\mathrm{O}$ atom. The end group $\mathrm{C}-\mathrm{OH}$ in the $\mathrm{PEO}$ chains was also identified at the peak of $1059 \mathrm{~cm}^{-1}$. From the spectra in Figure 14, no obvious difference in the peak positions was found between the two groups, which demonstrated that the ultrasonic treatment did not alter the chemical composition of the solid electrolyte. On the other hand, the ultrasonic treatment did not exert a negative influence on the chemical composition by causing scission or degradation of the polymer chains.

\subsection{Crystallinity}

Crystallinity is the degree of structural order in the electrolyte. In a crystalline part, the molecules are arranged in a regular, periodic manner, and thus the crystallinity has a big influence on the migration of $\mathrm{Li}^{+}$.

For a highly crystallized material, crystalline grains tend to be intact. The XRD peak is strong and sharp, and the full width at half maxima (FWHM) is close to the instrumental broadening limit. For a poorly crystallized material, crystalline grains are smaller and fewer, and the defects are also increased. The diffraction peaks are broad and weak. In this study, XRD tests were performed on the electrolytes of both groups. The results were analyzed by Jade 6.0, as shown in Figure 15 . As displayed in Figure 15, both spectra exhibited two low and broad peaks, indicating an amorphous phase. The $2 \theta$ values were $13.8^{\circ}$ and $19.8^{\circ}$, respectively, and the FWHM was $8^{\circ}$. At $19^{\circ}$ and $23^{\circ}$, sharp and narrow crystalline diffraction peaks appeared. Compared with the standard card, the diffraction peaks were the characteristic peaks of the PEO matrix. The simultaneous presences of the halo region and the crystalline peaks indicated the semi-crystalline nature of the PEO. The peak at $14.6^{\circ}$ was due to LiTFSI in the electrolytes. 


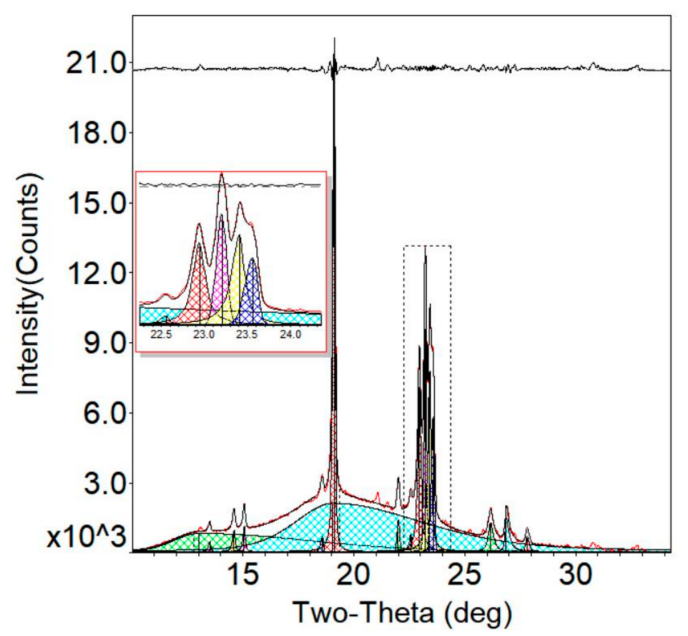

(a)

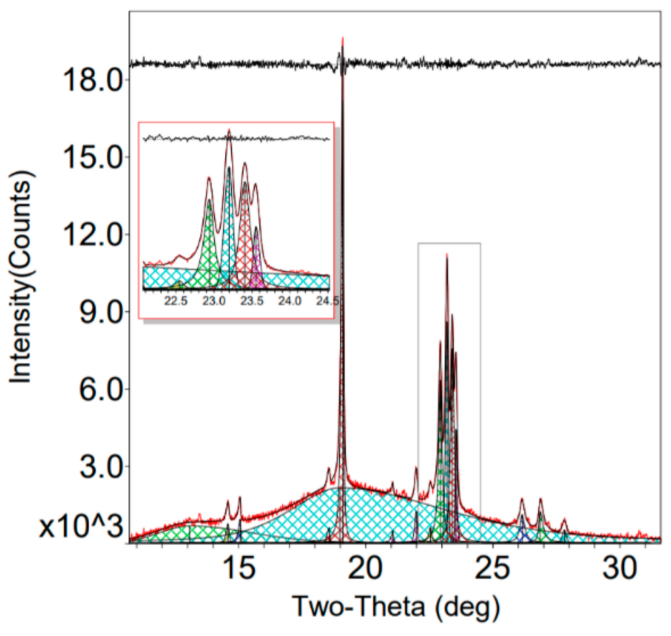

(b)

Figure 15. XRD analysis of the electrolytes from: (a) Group A, and (b) Group B.

As shown in Figure 15, the intensity of the PEO peaks was decreased for Group B due to the ultrasonic treatment, indicating a decrease in the PEO crystallinity and an increase in the PEO amorphous phase, which could enhance the ionic conductivity.

From the XRD pattern, the crystallinity can be calculated by:

$$
X_{c}=\frac{I_{c}}{I_{t}} \times 100 \%
$$

where, $X_{c}$ is the crystallinity, and $I_{c}$ is the summed intensity of the crystalline peaks, and $I_{t}$ is of the total intensity. The calculated results show that the crystallinity of Group A (without the ultrasonic treatment) was $36.1 \%$, and that of Group B (with the ultrasonic treatment) was $29.9 \%$. In addition, with the ultrasonic treatment, the intensity of the crystalline peak of the PEO was significantly reduced at $19^{\circ}$. This demonstrated that due to the ultrasonic vibration treatment, the crystallinity of the electrolyte membrane was significantly reduced. The amorphous phase increased, which was beneficial to the transport of the lithium ions. The decrease in the crystallinity resulted from the mechanical and thermal effects of the ultrasonic high-frequency vibration. The mechanical effect broke the PEO crystalline grains, which decreased the grain size but increased the grain boundary. The high-frequency vibration caused friction at the grain boundary, and heat was generated there. Thus, the grains were melted from the boundary. Due to the pulse mode of the ultrasonic treatment, the melting was slight and the solidifying was fast. The crystallization was suppressed under this condition. Therefore, the crystallinity was reduced, improving the ionic conductivity of the electrolyte. On the other hand, as shown in the test result in Section 3.1, the electrolyte of Group B lost the increased conductivity after it was annealed to room temperature from a molten state. The morphology modified by the ultrasonic treatment was rearranged during the annealing process, and thus the effect of the ultrasonic treatment was eliminated. Furthermore, the lower crystallinity reduced the electrolyte yield strength but also improved the plasticity. From the XRD pattern, the amorphous peak was at $13.712^{\circ}$ and $19.968^{\circ}$ for the electrolyte of Group A, while the peak was at $13.851^{\circ}$ and $20.268^{\circ}$ for the electrolyte of Group B. The slight right shift of the amorphous peak illustrated the larger diffraction angle, indicating a denser structure.

In the SPE, ethylene oxide (EO, the ether group) units have a high donor number for $\mathrm{Li}^{+}$and high chain flexibility, which are important for promoting ion transport. The ion hopping mechanism is dominant in the ion transport [24]. The migration of $\mathrm{Li}^{+}$from one site to the neighboring site is facilitated by the presence of vacancy defects, and therefore PEO crystallization is considered to be detrimental to ion transport due to the absence of vacancy in the area. According to the aforementioned 
analysis, the ultrasonic treatment brought large energy to the solid electrolyte through high-frequency vibration, which broke PEO grains and melted them with the frictional heat at the boundary. Due to the slight melting and fast solidifying produced by the pulse mode of the ultrasonic treatment, the crystallization was suppressed. Therefore, the treatment effectively reduced crystallinity of the PEO electrolyte membrane, which increased the migration channels of lithium ions. Smaller grains decreased the tortuosity effect as well, shortening the $\mathrm{Li}^{+}$transport path, and thus improved the ionic conductivity. Moreover, the ultrasonic treatment compressed the electrolyte, inducing the plastic deformation, and thus the solid electrolyte became tighter. The density of EO units was increased in the amorphous phase, providing multiple electron-donor coordination sites for the $\mathrm{Li}^{+}$. The ion hopping distance between donors was decreased, which facilitated the migration. As a result, the ion migration rate was increased, as shown in Figure 16. In addition, the mechanical performance of the electrolyte membrane was thus improved.

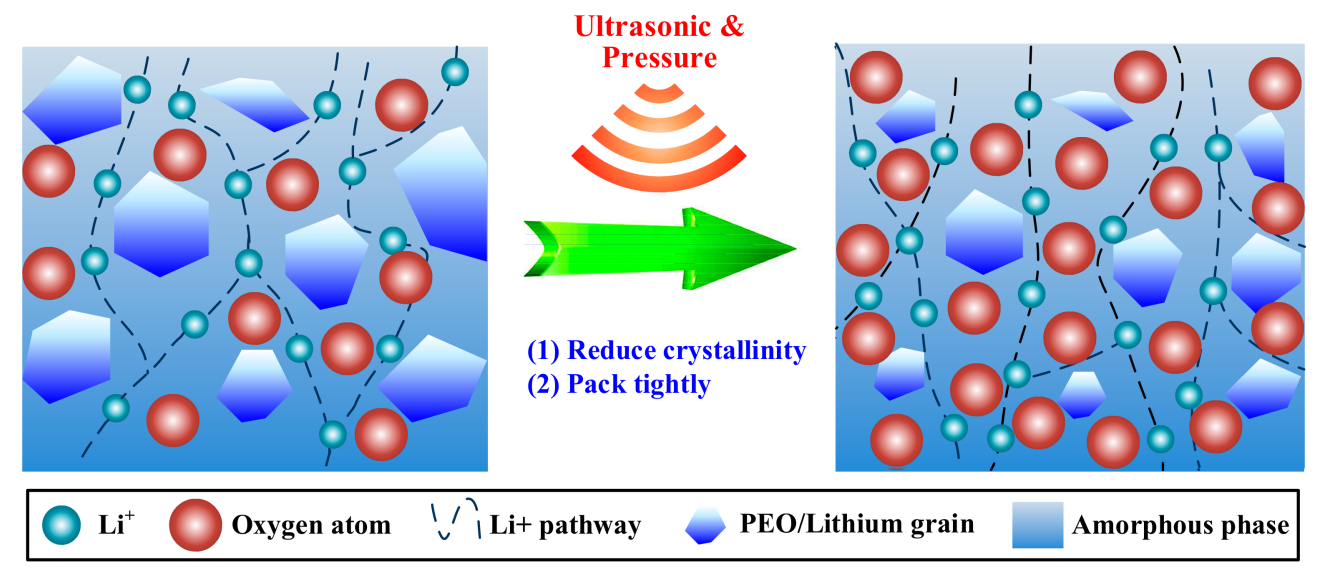

Figure 16. Ultrasonic promotion of ionic conductivity of the solid polymer electrolyte (SPE).

\section{Conclusions}

In this study, the ultrasonic vibration method was used to improve the performance of the PEO-based solid electrolyte. The power ultrasound was exerted on the electrolyte by a sandwich structure. The ultrasonic treatment reduced the crystallinity of the electrolyte, and increased the proportion of the amorphous phase. Through analysis of the performance and microstructure of the electrolyte, the effect of the ultrasonic treatment on the electrolyte was studied. The main conclusions are as follows:

1. The ultrasonic treatment significantly improved the ionic conductivity of the PEO-based electrolyte. The ionic conductivity was increased by $78 \%$. In addition, the ultrasonic treatment did not obviously affect the electronic conductivity. The prepared electrolyte had a wide electrochemical window, even at $80^{\circ} \mathrm{C}$.

2. The ultrasonic treatment broke PEO grains and melted them with the frictional heat at the boundary. Due to the slight melting and fast solidifying process, the crystallization was suppressed. The ultrasonic vibration effectively reduced the crystallinity by $6.2 \%$, which increased the migration channels of lithium ions. Smaller grains decreased the tortuosity effect as well, shortening the $\mathrm{Li}^{+}$ transport path, and thus improved the ionic conductivity.

3. The ultrasonic treatment compressed the electrolyte to produce plastic flow of the material, which made the electrolyte structure more compact. The density of EO units was thus increased in the amorphous phase, providing multiple electron-donor coordination sites for the $\mathrm{Li}^{+}$. The hopping distance of the ion between donors was decreased as well, which also facilitated the migration. 
4. The ultrasonic treatment did not alter the chemical composition and thermal stability of the solid electrolyte. The results show no negative effects produced, such as ultrasonic-induced bond break or thermal degradation.

5. The ultrasonic treatment improved the mechanical property of the electrolyte. The plasticity was improved, though the yield strength was slightly reduced. The tensile strength and elongation were both increased significantly.

This study presented a physical method to promote the ionic conductivity of the solid electrolyte using ultrasonic vibration, providing a reference for the improvement of polymer based all-solid-state batteries.

Author Contributions: Conceptualization, methodology and formal analysis, H.W.; experiments design, data analysis and draft writing, X.C., C.Z. and W.D.; supervision and editing, H.G. and Y.C. All authors contributed to writing and correcting the manuscript. All authors have read and agreed to the published version of the manuscript.

Funding: Funding: This work was supported by the National Natural Science Foundation Council of China, grant numbers 51775398, 51805392; the 111 Project, grant number B17034; the Program for Innovative Research Team in University of Education Ministry, grant number IRT_17R83; the Natural Science Foundation of Hubei Province, grant number 2018CFB595; and the National innovation and entrepreneurship training program for college students, grant number S201910497019.

Conflicts of Interest: The authors declare no conflict of interest.

\section{References}

1. Wang, L.; Li, X.; Yang, W. Enhancement of electrochemical properties of hot-pressed poly(ethylene oxide)-based nanocomposite polymer electrolyte films for all-solid-state lithium polymer batteries. Electrochim. Acta 2010, 55, 1895-1899. [CrossRef]

2. Mueller, T.; Hautier, G.; Jain, A.; Ceder, G. Evaluation of tavorite-structured cathode materials for lithium-ion batteries using high-throughput computing. Chem. Mater. 2011, 23, 3854-3862. [CrossRef]

3. Fujimura, K.; Seko, A.; Koyama, Y.; Kuwabara, A.; Kishida, I.; Shitara, K.; Fisher, C.A.J.; Moriwake, H.; Tanaka, I. Accelerated materials design of lithium superionic conductors based on first-principles calculations and machine learning algorithms. Adv. Energy Mater. 2013, 3, 980-985. [CrossRef]

4. Wang, Y.; Richards, W.D.; Ong, S.P.; Miara, L.J.; Kim, J.C.; Mo, Y.; Ceder, G. Design principles for solid-state lithium superionic conductors. Nat. Mater. 2015, 14, 1026-1031. [CrossRef] [PubMed]

5. Hautier, G.; Jain, A.; Chen, H.; Moore, C.; Ong, S.P.; Ceder, G. Novel mixed polyanions lithium-ion battery cathode materials predicted by high-throughput ab initio computations. J. Mater. Chem. 2011, 21, 17147-17153. [CrossRef]

6. Hautier, G.; Fischer, C.; Ehrlacher, V.; Jain, A.; Ceder, G. Data mined ionic substitutions for the discovery of new compounds. Inorg. Chem. 2011, 50, 656-663. [CrossRef]

7. Jalem, R.; Aoyama, T.; Nakayama, M.; Nogami, M. Cheminform abstract: Multivariate method-assisted ab initio study of olivine-type limxo4 (main group $\mathrm{m}^{2+}-\mathrm{x}^{5+}$ and $\mathrm{m}^{3+}-\mathrm{x}^{4+}$ ) compositions as potential solid electrolytes. Cheminform 2012, 43. [CrossRef]

8. Lee, K.J.; Yi, E.J.; Kim, G.; Hwang, H. Synthesis of ceramic/polymer nanocomposite electrolytes for all-solid-state batteries. J. Nanosci. Nanotechnol. 2020, 20, 4494-4497. [CrossRef]

9. Chintapalli, S.; Frech, R. Effect of plasticizers on high molecular weight peo-licf_3so_3 complexes. Solid State Ion. 1996, 86, 341-346. [CrossRef]

10. Kumar, Y.; Hashmi, S.A.; Pandey, G.P. Lithium ion transport and ion-polymer interaction in peo based polymer electrolyte plasticized with ionic liquid. Solid State Ion. 2011, 201, 73-80. [CrossRef]

11. Scrosati, B.; Croce, F.; Panero, S. Progress in lithium polymer battery R\&D. J. Power Sources 2001, 100, 93-100. [CrossRef]

12. Tan, S.M.; Johan, M.R. Effects of $\mathrm{MnO}_{2}$ nano-particles on the conductivity of pmma-peo-liclo 4 ec polymer electrolytes. Ionics 2011, 17, 485-490. [CrossRef]

13. Nairn, K.; Forsyth, M.; Every, H.; Greville, M.; Macfarlane, D.R. Polymer-ceramic ion-conducting composites. Solid State Ion. 1996, 86, 589-593. [CrossRef] 
14. Yu, X.Y.; Xiao, M.; Wang, S.J.; Zhao, Q.Q.; Meng, Y.Z. Fabrication and characterization of peo/ppc polymer electrolyte for lithium-ion battery. J. Appl. Polym. Sci. 2010, 115, 2718-2722. [CrossRef]

15. Niitani, T.; Shimada, M.; Kawamura, K.; Dokko, K.; Rho, Y.H.; Kanamura, K. Synthesis of li[sup +] ion conductive peo-pst block copolymer electrolyte with microphase separation structure. Electrochem. Solid State Lett. 2005, 8, A385-A388. [CrossRef]

16. Harras, B.; Cole, K.C.; Vu-Khanh, T. Optimization of the ultrasonic welding of peek-carbon composites. J. Reinf. Plast. Compos. 1996, 15, 174-182. [CrossRef]

17. O'Shaughnessey, P.G. Modeling and experimental investigation of induction welding of thermoplastic composites and comparison with other welding processes. J. Compos. Mater. 2016, 50. [CrossRef]

18. Choi, J.; Isayev, A.I. Natural rubber/styrene butadiene rubber blends prepared by ultrasonically aided extrusion. J. Elastomers Plast. 2015, 47, 170-193. [CrossRef]

19. Isayev, A.; Chang, K.H. Novel ultrasonic process for in-situ copolymer formation and compatibilization of immiscible polymers. Polym. Eng. Sci. 2003, 43, 91-101. [CrossRef]

20. Bera, M.; Rana, A.; Chowdhuri, D.S.; Hazari, D. A new 3d silver(i) coordination polymer with octadentate diglycolate ligand having silver-silver bond. J. Inorg. Organomet. Polym. Mater. 2012, 22, 1074-1080. [CrossRef]

21. Rizzolo, R.H.; Walczyk, D.F. Ultrasonic consolidation of thermoplastic composite prepreg for automated fiber placement. J. Thermoplast. Compos. Mater. 2016, 29, 1480-1497. [CrossRef]

22. Fernandez Villegas, I.; Van Moorleghem, R. Ultrasonic welding of carbon/epoxy and carbon/peek composites through a pei thermoplastic coupling layer. Compos. Part A Appl. Sci. Manuf. 2018, 109, 75-83. [CrossRef]

23. Chu, Q.; Li, Y.; Xiao, J.; Huan, D.; Zhang, X. Processing and characterization of the thermoplastic composites manufactured by ultrasonic vibration-assisted automated fiber placement. J. Thermoplast. Compos. Mater. 2018, 31, 339-358. [CrossRef]

24. Li, X.; Cheng, S.; Zheng, Y.; Li, C.Y. Morphology control in semicrystalline solid polymer electrolytes for lithium batteries. Mol. Syst. Des. Eng. 2019, 4, 793-803. [CrossRef]

(C) 2020 by the authors. Licensee MDPI, Basel, Switzerland. This article is an open access article distributed under the terms and conditions of the Creative Commons Attribution (CC BY) license (http://creativecommons.org/licenses/by/4.0/). 